\title{
A Decision Model for Emergency Warehouse Location Based on a Novel Stochastic MCDA Method: Evidence from China
}

\author{
Junkang He, Chenpeng Feng, Dan Hu, and Liang Liang \\ School of Management, Hefei University of Technology, Hefei, Anhui 230009, China \\ Correspondence should be addressed to Chenpeng Feng; cpfeng@hfut.edu.cn
}

Received 11 June 2017; Revised 31 August 2017; Accepted 25 September 2017; Published 16 November 2017

Academic Editor: Qingling Zhang

Copyright (C) 2017 Junkang He et al. This is an open access article distributed under the Creative Commons Attribution License, which permits unrestricted use, distribution, and reproduction in any medium, provided the original work is properly cited.

\begin{abstract}
China is one of the disaster-prone countries in the world. Constructing a rapid and effective relief logistic system is important for disaster-responding at country level. Strategic prepositioning of emergency items, especially the decision of appropriate emergency warehouses location, has significant impacts on rapid disaster response to ensure sufficient relief supplies. The emergency warehouse location decision is a complex problem, where a wide variety of criteria need to be considered and the preference information of decision makers (DMs) may be imprecise or even absent. In this paper, we identify key effectiveness-oriented criteria used to evaluate the alternative emergency warehouse locations and make an attempt to propose a new multicriteria ranking method to solve the problem of inaccurate or uncertain weight information based on stochastic pairwise dominant relations and the pruning procedure of ELECTRE-II method. The proposed method extends the conventional ELECTRE-II method by incorporating inaccurate information and broadens its application to emergency warehouse location field. The feasibility and applicability of the proposed method are illustrated with a simulated example.
\end{abstract}

\section{Introduction}

In the last few decades, the frequency of disasters has rapidly increased [1]. China, with complex climatic and geographical conditions and fragile ecological environment, is vulnerable to natural disasters. Frequent occurrences of large-scale natural disasters in recent years have caused a series of serious damage incidents. For example, the Richter 7.1 Yushu earthquake in 2010 caused 2,968 deaths and 0.5 billion dollars of economic loss. What is more, in May 12, 2008, a Richter 8.0 earthquake that occurred in Wenchuan county, Sichuan province, caused 87,476 deaths and 85 billion dollars of economic loss. Statistics by Emergency Management Database show that natural disasters in China have resulted in 90 thousand deaths with more than 160 billion dollars' economic losses since 2006.

The abovementioned data show that damage by disasters is still tremendous, for which one of the reasons is lack of relief supplies and rapid logistics [2]. After a fatal natural disaster, the demand of emergency supplies, ranging from rescue apparatus, medical equipment, and first-aid medicines to food and water, would be vastly increased within a short time. Facing the complex and severe conditions of disasters, researchers deem that a rapid response and preparedness are a crucial process to disaster relief [3]. The objective of disaster response in the humanitarian relief chain is to rapidly provide relief to minimize human suffering and death [4] and preparedness increases the ability of relief organizations to mobilize relief supplies and deliver aid quickly [5]. Among the different forms of preparedness for disaster relief management, prepositioning of emergency warehouses is considered to be the best for maximizing the effectiveness of humanitarian aid supply chains [6]. Prepositioning in strategic locations around the world is a strategy that has been implemented by humanitarian relief organizations to improve their capacities in delivering sufficient relief aid within a relatively short timeframe and with improved mobilization [5]. Fortunately, Chinese government pays increasing attention to strategic emergency prepositioning to rescue disaster victims as soon as possible by delivering sufficient relief supplies effectively. Issued on August 31, 2015, the Guidance on Strengthening the Construction of Natural Disasters Relief Supplies Reserve System points out that China will establish 5-level emergency warehouses to ensure that the victim of disasters receives 
basic necessary relief items within 12 hours after disasters break out. According to the Guidance, Ministry of Civil Affairs prepares to establish 24 central relief supplies warehouses in the cities around the country (i.e., national level warehouses), including Beijing, Tianjin, Shenyang, Harbin, Hefei, and other major cities. In addition, local authorities from province to county, especially in multihazard and easy-prone areas, build up the corresponding level relief supplies warehouses in terms of the actual conditions and the population distribution. Warehouse location decisions affect the performance of relief operations, since the number and location of the prepositioning warehouses and the amount of relief supply stocks held therein directly affect the response time and costs incurred throughout the humanitarian relief chain [5]. In this context, the selection of the warehouse location is considered to be rather significant for a rapid response in emergency relief [7].

Our research aims to provide an effective decision model for selecting the emergency prepositioning warehouse location. We provide a flexible and systematic framework, which details the guidelines for evaluating and selecting emergency prepositioning warehouse location for decision makers (DMs). Meanwhile we select the key criteria which are strongly believed to correctly evaluate the alternative locations based on theoretical results and practical conditions. The identified criteria, which are more relevant with the characteristics of locating the prepositioning warehouse than other effectiveness-oriented criteria, will reduce the decision risk and increase the chances of achieving successful completion. Furthermore, we propose the stochastic multicriteria decision analysis (MCDA) method to deal with the problem with less preference information. The proposed method will help DMs to make the most appropriate choice according to constructing stochastic pairwise dominant relation and utilizing the pruning procedures of ELECTRE-II method.

For supporting the decision of locating prepositioning warehouse, the decision-making framework is decomposed into five main phases as shown in the following:

(1) Determine the alternatives.

(2) Clarify the criteria.

(3) Collect and measure the criteria values.

(4) Choose and implement the proposed approach.

(5) Determine the preferred alternative.

Generally, the procedures of determining the preferred place for locating prepositioning warehouse proceed straightforwardly through all phases.

The remainder of this paper is organized as follows: Section 2 reviews the relevant literature. Section 3 identifies the key criteria used in ranking the alternatives of emergency prepositioning. In Section 4 the details of the proposed decision-aid method are presented. Section 5 depicts an application to solve a warehouse location selection problem. Finally, some conclusions are presented in the last section.

\section{Literature Review}

In this section, we briefly review the two major related literatures: the warehouse location problem in disaster relief by MCDA and developed stochastic ELECTRE methods.

2.1. Warehouse Location Problem. Disaster relief logistics management is categorized into three phases: preparation, immediate response, and reconstruction [8]. The objective of preparation is to improve rapid response facilities so as to deliver in emergency situations timely [9]. Hence, preparation is considered as an important phase in the relief process where the pressure of time in relief chain is "life and death" rather than a question of money [10]. A number of problems have been investigated for the preparation phase; one of the key issues is the warehouse location problem. In this subsection, warehouse location problem applied with MCDA methods will be presented because it is the main research tool used in the current study.

MCDA is applied to support DMs in the process of making a choice among alternatives. It has been widely applied in selecting location [11-14]. The selection of a preferred warehouse location among alternatives is a typical MCDA problem [15], which is necessary to take into consideration various criteria. MCDA methods offer the tools to provide the DMs with the analysis to be able to make better decision when building the prepositioning warehouse.

Alberto has addressed the relocation problem, and a decision-support approach has been developed based on the analytic hierarchy process to select the most preferred site as well as its relative advantage over other candidate sites [16]. The approach has been shown to be capable of handling multiple conflicting objectives such as the maximization of the logistic service to the customers and the minimization of cost. It can be a valid aid to analyzing the various tradeoffs among the competing alternatives and evaluating the implications of strategic relocation decisions. Kuo et al. present an effective fuzzy multicriteria analysis method based on the incorporated efficient fuzzy model and concepts of positive ideal and negative ideal points to solve decisionmaking problems with multijudges in the real-life environment, where judges are allowed to use fuzzy sets to evaluate the performance of alternatives and the importance of criteria [17]. This method efficiently grasps the ambiguity existing in available information as well as the essential fuzziness in human judgment and preference, and it always produced satisfactory results for all the cases examined in terms of rationality and discriminatory ability. Korpela and Tuominen make an attempt to apply an integrated approach to support the decision of selecting the location of the warehouse where both tangible and intangible criteria can be taken into account by using an analytic hierarchy process-based decision aid [18]. The proposed approach to the warehouse site selection process enables decision makers to base the final choice on the overall cost/service-effectiveness of the potential warehouses. Handayani et al. used AHP and fuzzyTOPSIS to select the criteria and subcriteria in determining the location of a catastrophic disaster logistics warehouse in Sleman, Yogyakarta, while fuzzy-TOPSIS used to rank the 
final location, and choose the preferred warehouse location among many alternatives [19]. Özcan et al. draw a comparison among AHP, TOPSIS, ELECTRE, and Grey Theory in terms of main characteristic of decision theory; thus advantages and disadvantages of these methodologies are offered [20]. Later, the application of these methodologies on the warehouse selection problem, which is one of the main topics of logistics management that has a wide range of applications with multicriteria decision-making methodologies, is presented as a case study which is characterized in retail sector that maintains high uncertainty and product variety and then how to choose preferred alternative warehouse location has been shown. Kayikci focuses on presenting a scientific method with multicriteria and multilevel decision-making aspects to solve a location selection problem for intermodal freight logistics centers [21]. A combination of fuzzy AHP and ANN techniques was applied to find a solution of location decision problem within given alternatives of selection. This hybrid model can give better results for decision-making problems while the fuzzy AHP was used to determine most important weight factors and ANN, to select the preferred location.

The above literature confirms that MCDA methods are applicable and practical in study of the warehouse location decision. However, it is difficult to figure out whether the MCDA method is used for humanitarian relief purposes. And these studies only meet business-focused criteria considered for selection of warehouse location. However, practically the main objective of relief supplies prepositioning is to have a rapid response to emergency relief; therefore efficiency is accorded higher priority than cost effectiveness in the problem. What is more, traditional methods for warehouse location selection problem tend to be less effective in dealing with the problem since the preference information of DM is usually imprecise or even absent. It is essential to capture the critical aspects of the warehouse location problem in humanitarian relief and identify the factors of locating a relief supplies warehouse.

2.2. ELECTRE Methods. The first ELECTRE method was presented by Benayoun et al. [22]. In the last few decades, the improved ELECTRE methods such as the ELECTRE-II, ELECTRE-III, ELECTRE-IV, ELECTRE-TRI, and ELECTRE-IS [23-27] have been proposed in succession and widely applied in certain types of real world problems including engineering, economics, management, and environment. Although several decades have passed since the birth of the first ELECTRE method, research on ELECTRE family method is still active and evolving today [28-31]. All ELECTRE methods belong to the family of outranking methods [32], one of the classic families of methods within MCDA. Each method consists of two phases: aggregation and exploitation [33].

It is important to point out that ELECTRE-II was the first method to use a technique based on the construction of an embedded outranking relations sequence [34], and the exploitation procedure of ELECTRE-II has guiding significance to this paper. Comparing with traditional MCDA methods, ELECTRE-II method, which has clear sequence logic, requires less cognitive effort and less preference information from the DM. ELECTRE-III was designed to improve ELECTRE-II and thus deal with inaccurate, imprecise, uncertain, or ill-determination of data. This purpose was actually achieved, and ELECTRE-III was applied with success during the last two decades on a broad range of real-life applications [34]. Nevertheless, for some users, these methods are very difficult to understand and apply [35], and they are rather limited and intricate because they require a number of parameters (like thresholds) set by DM and explicit criteria weight information. Obviously, the selection of each parameter may lead to the uncertainty of the results, especially in a lack of decision makers' preference information condition. And the criteria weight, however, is uncertain in many practical applications, which may result in infeasibility of the method. In these cases, it is difficult to guarantee the stability and accuracy of alternatives sort.

Hence, many developed stochastic ELECTRE methods have been investigated to solve the problem with inaccurate or uncertain preference information. Tervonen et al. have presented a novel method of inverse weight-space analysis for ELECTRE-III [36]. The analysis is based on a modified version of the Stochastic Multicriteria Acceptability Analysis (SMAA), which is a family of decision-support methods to aid DMs in discrete decision-making problems. Their method allows the weights of ELECTRE-III to be of arbitrary type: no deterministic weights are required and weight information is provided as weight intervals; it has numerous advantages, especially in the context of MCDA with multiple DMs, because the weights can be determined as intervals which contain the preferences of all DMs. SMAA- 3 method is a decision aid which does not require any explicit preference information from the DMs during the decision-making procedure [37]. It is a variant of the original SMAA that applies, instead of the utility function, ELECTRE-type pseudocriteria and maxi-min choice procedure in the analysis. The uncertainty of the basic data is modeled using ELECTRE-III-type pseudocriteria with preference and indifference thresholds. It should be noted that SMAA-3 was found to be quite unstable with respect to the indifference threshold [38]. A drawback of the SMAA-3 method is that it ignores discordance indices and only ranks the alternatives based on their concordance indices [39]. Tervonen et al. propose a new method, SMAATRI, which is based on SMAA and developed for parameter stability analysis of ELECTRE-TRI [40]. It allows ELECTRETRI to be used with imprecise, arbitrarily distributed values for weights and the lambda cutting level. The method consists of analyzing through Monte Carlo simulation finite spaces of arbitrarily distributed parameter values in order to provide DMs with values characterizing the problem. The SMAA-TRI analysis results in category acceptability indices for all pairs of actions and categories, and these can be used to analyze the stability of the parameters. By visualizing the category acceptability indices with stacked columns the uncertainty related to each assignment decision can be presented to the DMs in a comprehensible way. Zhou et al. integrate SMAA with ELECTRE to deal with gray stochastic MCDA problem, with criteria values being extended gray random variables [39]. The extended gray random variables accommodate the stochastic decision-making environment and exhibit a 
powerful capacity to express uncertain information. The proposed approach provides recommendations for alternatives based on uncertain preference information, and it can effectively solve the stochastic problems with imprecise, partial, missing, or conflicting weight information.

These literatures provide significant and available stochastic methods to solve the MCDA problem that weight information is imprecise or absent. In the process of constructing warehouse for emergency relief, often, the DMs (usually government officials) can only give partial preference information, because they are unwilling to shoulder the decision risk owing to expressing preferences too much or sometimes possessing insufficient decision cognition and relative knowledge. However, there is no literature applying stochastic MCDA method in warehouse location problem for humanitarian relief purpose. It should be noted that, instead of giving direct answers to the decision-making problem, SMAA methods are based on inversely analyzing and characterizing the problem, leaving the final decision for DMs [36]. Sometimes DMs may just want unambiguous and straightforward ranking results when making certain hard decisions or when they are unwilling to take more decision risks. In this study, we attempt to address the concern with proposing a new stochastic way access to a direct and explicit ranking result when DMs' preference information is uncertain, imprecise, and/or missing.

\section{Determining the Criteria}

In order to determine the preferred choice of the alternative regions for constructing the prepositioning warehouse, one has to determine key factors on which the evaluation of the alternatives is based. After analyzing the literature ([7, 15, $16,18]$, etc.), certain variations of relative factors taken into consideration in the evaluation are identified, such as start-up cost, traffic condition, capacity, and climate. In addition, we have consulted some relative experts and government officials to define factors which they perceived to be significant. With these efforts, dozens of factors were obtained.

Yang et al. studied the construction of reserve network for China Red Cross and proposed five principles to screen the specific factors impacting the preferred warehouse location selection in prevention of earthquake [2]:

(i) The warehouses should be selected in the safe places to avoid being damaged.

(ii) The storage capacity should be large enough to accommodate relief supplies.

(iii) Traffic should be smooth around the warehouse to ensure rapid response to emergency relief (if earthquake breaks out).

(iv) Based on the principle of equity, the warehouse should be sited as close to all possible disaster areas as possible rather than just considering one or two important provinces.

(v) The selection of warehouses near to earthquake-prone areas with large population should be the priority.

Although the five principles are proposed to choose the preferred warehouses by China Red Cross in prevention for earthquake, these can also be used for other natural disasters. According to the factors we identified, the five principles and actual conditions in implementing the program, finally, we screen five criteria to evaluate the alternatives of locating prepositioning warehouse as follows:

(1) Traffic condition

(2) Stock holding capacity

(3) Surrounding environment for reserving relief supplies

(4) Distance to disaster-prone area

(5) Cost.

The definition and measurement for each criterion are as follows: Traffic condition means the convenience degree of personnel or material exchanges between disaster region and outside. It directly determines the efficiency and effectiveness of emergency relief. Thus, it is the factor that must be considered in the selection of emergency prepositioning warehouse. Traffic condition is measured by a five-point ratio scale based on the degree of the transport infrastructure containing not only the quantity, but also the quality of the roads. The value 5 represents the best condition to the transportation while the value 1 represents the worst one.

Particularly, each alternative location does not have the same capacity caused by certain practical factors, such as topography of the region and the constraint of land use. Sufficient capacity for reserving relief supplies is significant for emergency relief after disaster occurred. We measure stock holding capacity as the effective storage area to accommodate relief supplies; the greater the area, the better the warehouse.

Surrounding environment represents the basic situation of the natural nonhuman factors in the warehouse region. It is a manifestation of the security of the reserve warehouse and also the guarantee of smooth operation of the emergency rescue work. For instance, the prepositioning warehouse cannot be established in disaster-prone area. The surrounding environment also includes the meteorological conditions which may cause bad influences to some reserved supplies. For measuring this criterion, we also use a fivepoint ratio scale based on the degree of geographical and meteorological conditions. The value 5 represents the best condition to reserve the supplies, and correspondingly the value 1 represents the worst one.

Distance to disaster-prone area is also a critical factor for a rapid response to emergency relief. It is measured as the average vehicle mileage on the road from the prepositioning warehouse to the disaster-prone area.

Cost means the consumption of manpower, material, and financial resources in the process of warehouse construction and administration; it reflects the feasibility and economy of the relief supplies warehouse. It is essential to identify these costs as different alternatives may have different construction and administration costs. In reality, cost is measured based on a series of estimations in variations of aspects from relative experts.

For the specifics of emergency prepositioning, efficiency is considered to be more significant than effectiveness; thus, traffic condition and surrounding environment have empirically higher priority than cost when evaluating the location of relief supplies warehouse. 


\section{Methodology}

In the previous literature, the stochastic MCDA methods have been widely investigated to solve the problem of inaccurate or uncertain preference information, which have been considered as an effective decision tool. These methods provide a significant and available stochastic idea to solve the problem when preference information is partially known or absent. The concept of stochastic pairwise dominant relation of our method derives from the stochastic idea of these literatures. Comprehensively, we propose a stochastic MCDA approach considering both the stochastic idea and the pruning procedures of ELECTRE-II method.

4.1. Preliminaries. Consider a discrete set of alternatives $X=$ $\left\{X_{1}, X_{2}, \ldots, X_{m}\right\}$ and $C=\left\{C_{1}, C_{2}, \ldots, C_{n}\right\}$ is a set of $n$ criteria with the weight vector $W=\left(w_{1}, w_{2}, \ldots, w_{n}\right)^{T}$, where $w_{j}>0$ and $\sum_{j=1}^{n} w_{j}=1$. Let $Y=\left(y_{i j}\right)_{m \times n}$ be the original decision matrix, where $y_{i j}$ denotes the criteria value that the $i$ th alternative $X_{i} \in X$ can take with respect to criteria $C_{j} \in C$. As the difference in dimension among various criteria may affect the results of the overall evaluation, the criteria need to be dimensionless. Maximal and minimal criteria values are chosen to scale $Y=\left(y_{i j}\right)_{m \times n}$ into a normalization matrix $R=\left(r_{i j}\right)_{m \times n}$; that is,

$$
\begin{aligned}
& r_{i j}=\frac{\left(y_{i j}-y_{j}^{\min }\right)}{\left(y_{j}^{\max }-y_{j}^{\min }\right)}, \\
& r_{i j}=\frac{\left(y_{j}^{\max }-y_{i j}\right)}{\left(y_{j}^{\max }-y_{j}^{\min }\right)} .
\end{aligned}
$$

Equation (1) is used as the normalization scheme for benefit criteria, while nonbenefit criteria are normalized by using (2).

The ELECTRE-II method employs a technique based on the concept of outranking relationship, which includes two phases: the construction of outranking relation and the pruning procedure of the relation [34]. The outranking relation is constructed to make comparison in a comprehensive way between each pair of actions.

Step 1. The outranking relation is constructed to make comparison in a comprehensive way between each pair of actions. When there are reasons for believing that " $X_{i}$ is at least as good as $X_{k}$ " $\left(X_{i}, X_{k} \in X\right)$, the level of $X_{i}$ is higher than $X_{k}$; this is so-called outranking method. The important thing to note is that outranking relation is built on the basic that DMs are willing to bear the risks when they admit " $X_{i}$ is at least as good as $X_{k}$." The construction of an outranking relation is based on two major concepts [34].

Concordance. For an outranking $X_{i} O X_{k}$ to be validated, a sufficient majority of criteria should be in favor of this assertion.

Nondiscordance. When the concordance condition holds, none of the criteria in the minority should oppose too strongly to the assertion $X_{i} O X_{k}$.
Before defining the concord condition, let $J^{+}$represent those criteria for which $X_{i}$ is strictly preferred to $X_{k}$. Similarly let $J^{=}$represent those criteria for which $X_{i}$ is preferred as good as $X_{k}$ and $J^{-}$represent those criteria for which $X_{k}$ is preferred $X_{i}$. The criteria are divided into three subsets:

$$
\begin{aligned}
& J^{+}\left(X_{i}, X_{k}\right)=\left\{j \mid 1 \leq j \leq n, y_{i j}>y_{k j}\right\} ; \\
& J^{=}\left(X_{i}, X_{k}\right)=\left\{j \mid 1 \leq j \leq n, y_{i j}=y_{k j}\right\} ; \\
& J^{-}\left(X_{i}, X_{k}\right)=\left\{j \mid 1 \leq j \leq n, y_{i j}<y_{k j}\right\} .
\end{aligned}
$$

Then define

$$
\begin{aligned}
& W^{+}=\sum w_{j}, \quad j \in J^{+} ; \\
& W^{=}=\sum w_{j}, \quad j \in J^{=} ; \\
& W^{-}=\sum w_{j}, \quad j \in J^{-} .
\end{aligned}
$$

And finally the concord condition is defined:

$$
I_{i k}=\frac{\sum_{j \in J^{+}\left(X_{i}, X_{k}\right)} w_{j}+\sum_{j \in J^{=}\left(X_{i}, X_{k}\right)} w_{j}}{\sum_{j=1}^{n} w_{j}} .
$$

The nondiscord condition is introduced to represent veto situations so as to inspect the relative position of two compared alternatives on the value scales, for those criteria which are in discordance with the hypothesis that $X_{i}$ does not outrank $X_{k}$ [41]. The discordance condition $d_{i k}$ is defined as follows:

0 , if $y_{k j}<y_{i j}, \forall j$, and the goal is to maximize the criteria outcome

0 , if $y_{k j}>y_{i j}, \forall j$, and the goal is to minimize the criteria outcome.

$$
\text { Otherwise, } d_{i k}=\frac{\max \left(y_{k j}-y_{i j}\right)}{\delta},
$$

where $d_{i k}$ is the set of criteria for which $X_{i}$ is worse than $X_{k} . \delta$ is the maximum difference on a particular criterion [42]. As discordance and concordance conditions have been defined, it is possible to define the outranking procedure for ELECTRE-II. The outranking procedure consists of constructing two extreme relationships: a strong relationship $\mathrm{O}_{s}$ and a weak relationship $O_{w}$. In order to define $O_{s}$ and $O_{w}$, firstly determine three thresholds (high, medium, and low), respectively, called $\alpha^{-}, \alpha^{0}$, and $\alpha^{*}$, and $0 \leq \alpha^{-} \leq \alpha^{0} \leq \alpha^{*} \leq 1$. Furthermore, Determine two values of $d$, and $0<d^{0}<d^{*}<$ 1. With these specifications, the strong relationship and the weak relationship are defined, respectively, as follows:

$$
\begin{aligned}
& X_{i} O_{s} X_{k} \Longleftrightarrow\left\{\begin{array}{lll}
I_{i k} \geq \alpha^{*} & I_{i k} \geq \alpha^{0} \\
d_{i k} \leq d^{*} & \text { Or } & d_{i k} \leq d^{0} \\
W^{+} \geq W^{-} & W^{+} \geq W^{-} ;
\end{array}\right. \\
& X_{i} O_{w} X_{k} \Longleftrightarrow\left\{\begin{array}{l}
I_{i k} \geq \alpha^{-} \\
d_{i k} \leq d^{*} \\
W^{+} \geq W^{-} .
\end{array}\right.
\end{aligned}
$$


As a result of the two pairwise relationships, the graphs can be constructed, one for the strong relationship and one for the weak relationship. These graphs can be used in an iterative pruning procedure to obtain the desired ranking of the alternatives.

Step 2. The pruning procedure is used to elaborate recommendations from the results obtained in the first phase. As a result of the two correlations, the strong digraph and the weak digraph can be constructed. Implementing the iterative pruning procedure based on the two digraphs, it contains an upward ranking and a downward ranking, and the procedure can be described as follows [43].

(1) Upward Raking. The nodes (representing alternatives) of the digraphs which have no precedent are called noninferiority nodes. The set of noninferiority nodes in the strong digraph and the weak digraph is denoted as $G_{s}$ and $G_{w}$, respectively. Define intersection set $C=G_{s} \cap G_{w}$, and then sequence them. The upward ranking procedure can be described as follows.

$$
\text { Let } G_{s}^{1}=G_{s} \text { and } G_{w}^{1}=G_{w} \text {. }
$$

Identify all noninferiority nodes. Determine $G_{s}^{1}, G_{w}^{1}$ and the intersection set $C^{1}$.

Delete the nodes of $C^{1}$ and all precedent branches related to these nodes in the two digraphs. The remaining strong graph and weak graph are, respectively, denoted as $G_{s}^{2}$ and $G_{w}^{2}$. Then determine the intersection of them, called $C^{2}$.

Delete the nodes of $C^{2}$ and all the precedent branches from these nodes in the remaining digraphs.

Repeat these steps above. Obtain $G_{s}^{k+1}, G_{w}^{k+1}$, and $C^{k+1}$ until the intersection is a null set. If an alternative node belongs to the $C^{r}$ set, it will receive the ranking $r$.

(2) Downward Ranking. Reverse all the directions of the arcs in $G_{s}$ and $G_{w}$; a rank of mirror of upward ranking which is called downward ranking would be obtained. According to the procedure of upward ranking, the mirrored downward ranking value $\operatorname{rank}^{0}\left(X_{i}\right)$ of every alternative can be obtained which is readjusted by setting

$$
\begin{aligned}
\operatorname{rank}^{-}\left(X_{i}\right) & =1+\operatorname{rank}^{*}-\operatorname{rank}^{0}\left(X_{i}\right), \\
\operatorname{rank}^{*} & =\max _{X_{i} \in X} \operatorname{rank}^{0}\left(X_{i}\right) .
\end{aligned}
$$

(3) Final Ranking. The final ranking value of each alternative would be obtained by using the following averaging function:

$$
\overline{\operatorname{rank}(X i)}=\frac{\operatorname{rank}^{+}\left(X_{i}\right)+\operatorname{rank}^{-}\left(X_{i}\right)}{2} .
$$

Finally, rank in a decreasing manner the values given by the averaging function. This process yields the final ranking.

4.2. Stochastic Pairwise Dominance Probability. The weight parameter $w$ characterizes DMs' preference concerning the relative importance of criteria [41]. For describing uncertain weight information systematically and distinctly, we introduce the concept of weight space first. Denote $W=$ $\left(w_{1}, w_{2}, \ldots, w_{n}\right)^{T}$ as a set of weight combinations and designate weight space by $\Omega(W)=\Omega\left(w_{1}, w_{2}, \ldots, w_{n}\right)$, which contains all possible feasible weight combinations. In an $n$ dimensional weight space, all feasible weight combinations construct a $(n-1)$-dimensional simplex [44]. If the weight information is completely unknown, it can be defined as $\Omega(W)=\left\{w_{j}>0, \sum_{j=1}^{n} w_{j}=1\right\}$. Often, the following types of restriction on the weight space [45] can be handled taking into account the DMs' known preferences, when they have some decision cognition and relative knowledge:

(1) Partial (or complete) ranking of criteria $\left(w_{j}>w_{k}\right.$, for some $j, k)$

(2) Intervals for weights $\left(w_{j} \in\left[w_{j}^{\min }, w_{j}^{\max }\right]\right)$

(3) Intervals for weight ratios (trade-offs) $\left(w_{j} / w_{k} \in\right.$ $\left.\left[w_{j}^{\min }, \mathrm{w}_{j}^{\max }\right]\right)$

(4) Linear inequality constraints for weights $(A w \leq c)$

(5) Nonlinear inequality constraints for weights $(g(w) \leq$ $0)$.

For two given alternatives $f$ and $g$ under the weight space $\Omega(w)$, the probability of $f$ dominating $g$, denoted by $P_{f g}$, is defined as

$$
P_{f g}=P\left(\sum_{j=1}^{n} r_{f j} w_{j} \geq \sum_{j=1}^{n} r_{g j} w_{j}\right) .
$$

Obviously, the greater the dominant probability, the stronger the dominant relation. Specifically, if $P_{f g}>0.5$, then we declare that $f$ weakly dominates $g$ and vice versa. If $P_{f g}=$ 1 , it means $f$ dominates $g$ strictly, no matter the situation. If $P_{f g}=0$, it means $f$ dominates $g$ under no condition.

To measure the strong dominant relation, we denote $\theta$ $(0.5<\theta<1)$ as the critical value of strength given by the DM. For each pair of alternatives $f$ and $g$ in the alternatives set $X$, if $P_{f g} \geq \theta$ exists, then $f$ has strong dominant relation on $g$, denoted by $f>_{s} g$; if $0.5 \leq P_{f g}<\theta$, then $f>_{w} g$ is recognized. It is obvious that the greater the critical value, correspondingly, the higher the requirements of the strong dominant relation. However, the risk of decision-making is irrelevant to the change. Actually, the role of the strength critical value $\theta$ is to divide the set of dominant relation into two subsets (i.e., the set of strong/weak dominant relation) in order to generate a specific rank-order and avoid the appearance of the same ranking. Therefore, the selection of the parameter $\theta$ should be neither too large nor too small. We suggest that the selected parameter is appropriate when the amounts of element in each subset are roughly equivalent.

4.3. The Proposed Stochastic MCDA Method. For a lowdimensional weight space, the dominant probability can be directly calculated. For example, in a two-dimensional weight space (i.e., only two criteria in a MCDA problem), the weight space is a line segment in geometry and the dominant 


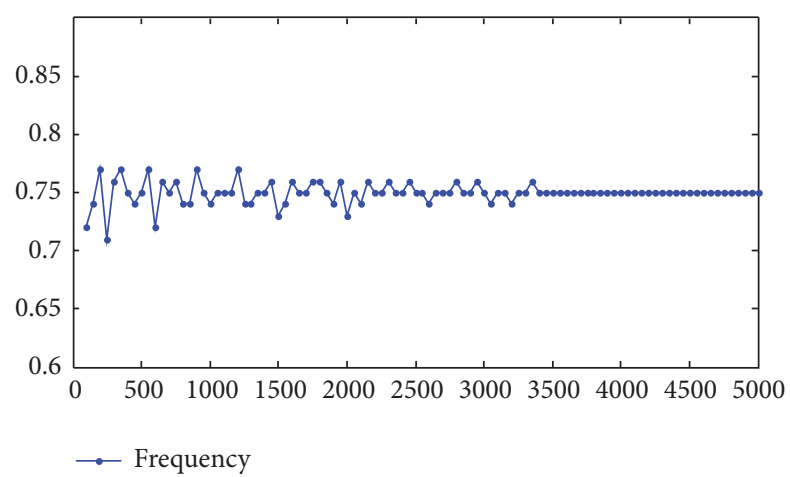

Figure 1: Convergence of the dominant probability in Monte Carlo simulation.

probability can be obtained by calculating the following formulation:

$$
P_{f g}=P\left(\left(r_{f 1}-r_{g 1}\right) w_{1}+\left(r_{f 2}-r_{g 2}\right) w_{2} \geq 0\right)=\frac{l_{d}}{l_{0}},
$$

where $l_{d}$ is the length of the part that $f$ dominates $g$ and $l_{0}$ is the length of the whole line segment. In a three-dimensional weight space, the space is a plane in geometry and the dominant probability can be calculated by

$$
\begin{aligned}
P_{f g} & =P\left(\left(r_{f 1}-r_{g 1}\right) w_{1}+\left(r_{f 2}-r_{g 2}\right) w_{2}\right. \\
& \left.+\left(r_{f 3}-r_{g 3}\right) w_{3} \geq 0\right)=\frac{S_{d}}{S_{0}},
\end{aligned}
$$

where $S_{d}$ is the area of the part that $f$ dominates $g$ and $S_{0}$ is the area of the whole plane. Unfortunately, there are usually at least 5 criteria in locating warehouse problems (i.e., the weight space is a multidimensional hyperplane). As a result, the analytic solutions of pairwise dominant probability will be difficult to obtain. Instead, the Monte Carlo simulation approach can be utilized to calculate pairwise dominant probability for high-dimensional situation.

To demonstrate the feasibility of this approach, we consider a simple numerical example involving 2 alternatives (denoted as $A$ and $B$ ) with 4 criteria. Let $A=(4,6,5,5)$ and $B=(5,4,3,6)$. Similar to the treatment presented in Lahdelma et al. [44], we use a uniform, random distribution of weight space when computing the dominant probability. And we argue that, without prior knowledge about valuations of the DM, the criteria weight space is thus $\Omega(w)=\left\{0 \leq w_{j} \leq\right.$ $\left.1, \sum_{j=1}^{4} w_{j}=1, j=1,2,3,4\right\}$. The dominant probabilities change relative to the times of Monte Carlo simulation is shown in Figure 1, and the result indicates that the dominant frequency converges to the value of 0.75 after more than about 3,500 simulations. Thus, the pairwise dominant probability is 0.75 .

These dominant probabilities of each pair of alternatives form a matrix $P$, denoted as $P=\left[P_{a b}\right]_{m \times m}, a, b=$ $1,2, \ldots, m$. Particularly, the elements on the diagonal are equal to 1 ; that is, $P_{a a}=1, \forall 1 \leq a \leq m$. Once the matrix has been completed through a series of calculations of pairwise dominant probabilities, the set of strong dominant relation $\left(Q_{s}\right)$ and the set of weak dominant relation $\left(Q_{w}\right)$ are determined by comparing with the strength critical value $\theta$ given by the DM, respectively. Based on the two sets, we can construct strong/weak dominant digraph. The procedure by which we sequence the alternatives by using the two digraphs seems like that stated in Step 2 of the ELECTRE-II method.

According to the abovementioned analysis, the procedure of the proposed stochastic MCDA method is implemented as follows.

Step 1 (form the warehouse location problem). Specify the criteria weights space based on the DMs' known preferences and standardize the original criteria values.

Step 2 (obtain the sets of $Q_{s}$ and $Q_{w}$ ). Obtain the set of strong/weak dominant relation by calculating pairwise dominant probability and comparing with the strength critical value $\theta$.

Step 3 (construct the two digraphs). As a result of the two dominant correlations, construct the strong digraph and the weak digraph and sequence all alternatives according to the digraphs. By implementing the iterative pruning procedure based on the two digraphs, it contains an upward and a downward ranking.

These operations in upward ranking, downward ranking, and calculating the ranking value are similar to those in Step 2 of ELECTRE-II stated in Section 4, but the outranking relation is replaced by the dominant relation. Finally, the ranking results can be obtained for each alternative according to procedures of the proposed approach.

\section{Illustrative Example}

In this section, we apply the proposed method to deal with a simulated selection problem of relief supplies warehouse, in which the DM has to choose among six alternative locations evaluated on five criteria: traffic condition, stock holding capacity, surrounding environment, distance, and cost. The criteria values of the six locations evaluated on the five criteria are represented in Table 1 (the data is accordant with practical circumstance as far as possible based on our preliminary investigation). According to the proposed method, the procedures implemented to support the decision of selecting the prepositioning warehouse location are as follows.

Step 1. Standardize original criteria values. The normalization criteria values obtained are shown in Table 2. In this example, it is assumed that the DM's preference information is missing so that the criteria order or other constraints of weight space could not be determined via the DM. However, as preceding arguments, traffic condition and surrounding environment are considered as higher priority than cost when determining the location of relief supplies warehouse. Thus, the weight space is finally defined as $\Omega(w)=\left\{w_{j}>0, w_{1}>\right.$ 
TABLE 1: Criteria values of alternatives.

\begin{tabular}{|c|c|c|c|c|c|}
\hline Alternative & Traffic condition & Capacity $\left(\mathrm{m}^{2}\right)$ & Surrounding environment & Distance $(\mathrm{km})$ & Cost (million RMB) \\
\hline$X_{1}$ & 3.2 & 10640 & 4.5 & 214 & 37.6 \\
\hline$X_{2}$ & 3.3 & 13000 & 3.6 & 202 & 29.6 \\
\hline$X_{3}$ & 2.0 & 9280 & 3.0 & 227 & 60.0 \\
\hline$X_{4}$ & 3.8 & 12000 & 4.8 & 175 & 34.0 \\
\hline$X_{5}$ & 3.5 & 11500 & 4.0 & 194 & 34.0 \\
\hline$X_{6}$ & 4.2 & 5850 & 3.9 & 150 & 21.0 \\
\hline
\end{tabular}

TABLE 2: Normalized criteria values of alternatives.

\begin{tabular}{|c|c|c|c|c|c|}
\hline Alternative & Traffic condition & Capacity & Surrounding environment & Distance & Cost \\
\hline$X_{1}$ & 0.55 & 0.67 & 0.83 & 0.17 & 0.57 \\
\hline$X_{2}$ & 0.59 & 1.00 & 0.33 & 0.32 & 0.78 \\
\hline$X_{3}$ & 0.00 & 0.48 & 0.00 & 0.00 & 0.00 \\
\hline$X_{4}$ & 0.82 & 0.86 & 1.00 & 0.68 & 0.67 \\
\hline$X_{5}$ & 0.68 & 0.79 & 0.56 & 0.43 & 0.67 \\
\hline$X_{6}$ & 1.00 & 0.00 & 0.50 & 1.00 & 1.00 \\
\hline
\end{tabular}

TABLE 3: Pairwise dominant probability matrix.

\begin{tabular}{ccccccc}
\hline & $X_{1}$ & $X_{2}$ & $X_{3}$ & $X_{4}$ & $X_{5}$ & $X_{6}$ \\
\hline$X_{1}$ & 1 & 0.51 & 1 & 0 & 0.26 & 0.34 \\
$X_{2}$ & 0.49 & 1 & 1 & 0.01 & 0.16 & 0.28 \\
$X_{3}$ & 0 & 0 & 1 & 0 & 0 & 0.02 \\
$X_{4}$ & 1 & 0.99 & 1 & 1 & 1 & 0.81 \\
$X_{5}$ & 0.74 & 0.84 & 1 & 0 & 1 & 0.36 \\
$X_{6}$ & 0.66 & 0.72 & 0.98 & 0.29 & 0.64 & 1 \\
\hline
\end{tabular}

$\left.w_{5}, w_{3}>w_{5}, \sum_{j=1}^{5} w_{j}=1\right\}$ and used in a uniform, random distribution when computing the dominant probability.

Step 2. Calculate the dominant probability of each pair of alternative locations; the pairwise dominant probability matrix is shown in Table 3. According to the argument stated in Section 4.2, the appropriate strength critical value $\theta$ is considered to be 0.9 after observation. Combined with the elements which are greater than 0.5 in pairwise dominant probability matrix, the set of strong dominant relations $\left(Q_{s}\right)$ and the set of weak dominant relations $\left(Q_{w}\right)$ obtained are shown below, respectively:

$$
\begin{aligned}
& Q_{s}=\left\{X_{1} \succ_{s} X_{3} ; X_{2} \succ_{s} X_{3} ; X_{4} \succ_{s} X_{1} ; X_{4} \succ_{s} X_{2} ; X_{4}\right. \\
& \left.>_{s} X_{3} ; X_{4}>_{s} X_{5} ; X_{5}>_{s} X_{3} ; X_{6}>_{s} X_{3}\right\} \text {; } \\
& Q_{w}=\left\{X_{1}>_{w} X_{2} ; X_{4} \succ_{w} X_{6} ; X_{5}\right\rangle_{w} X_{1} ; X_{5} \\
& \left.>_{w} X_{2} ; X_{6}>_{w} X_{1} ; X_{6}>_{w} X_{2} ; X_{6}>_{w} X_{5}\right\} \text {. }
\end{aligned}
$$

Step 3. Based on the correlations represented by the elements in the two sets, we construct the strong/weak digraph and set them as initial digraphs, shown as Figures 2(a) and 2(b).

Implement the pruning procedure to sequence all the alternative locations in the two digraphs. For example, in the initial strong digraph, the alternatives $X_{4}$ and $X_{6}$ are
TABLE 4: Results of the pruning procedure.

\begin{tabular}{lcccccc}
\hline Items & $X_{1}$ & $X_{2}$ & $X_{3}$ & $X_{4}$ & $X_{5}$ & $X_{6}$ \\
\hline $\operatorname{rank}^{+}\left(X_{i}\right)$ & 4 & 5 & 6 & 1 & 3 & 2 \\
$\operatorname{rank}^{0}\left(X_{i}\right)$ & 2 & 1 & 1 & 5 & 3 & 4 \\
$\operatorname{rank}^{-}\left(X_{i}\right)$ & 4 & 5 & 5 & 1 & 3 & 2 \\
$\overline{\operatorname{rank}\left(X_{i}\right)}$ & 4 & 5 & 5.5 & 1 & 3 & 2 \\
\hline
\end{tabular}

noninferiority nodes when no precedent branches existed. Thus, the set of noninferiority nodes in the initial upward strong digraph is $G_{s}^{1}=\left\{X_{4} ; X_{6}\right\}$. Similarly, $G_{w}^{1}=\left\{X_{4}\right\}$ can be obtained in the initial upward weak digraph. The intersection of $G_{s}^{1}$ and $G_{w}^{1}$ is determined as $C_{1}=G_{s}^{1} \cap G_{w}^{1}=\left\{X_{4}\right\}$. Thus, the ranking of $X_{4}$ is 1 in the upward ranking procedure. Next, delete the nodes in intersection $C_{1}$ (i.e., $X_{4}$ ) and all the branches related to the node in initial upward strong/weak digraph. In what follows, repeat the same operation until the intersection is null. Procedure of downward ranking is similar to upward ranking; reverse all the arrow directions in the initial upward digraph and implement the pruning procedure. Finally, the upward ranking values $\operatorname{rank}^{+}\left(X_{i}\right)$, the mirrored downward ranking values $\operatorname{rank}^{0}\left(X_{i}\right)$, the results of the real downward ranking values $\operatorname{rank}^{-}\left(X_{i}\right)$, and the final ranking values $\overline{\operatorname{rank}\left(X_{i}\right)}$ calculated by (8) and (9) are all shown in Table 4.

According to the ranking results in Table 4, it can be indicated that $X_{4}$ is the best befitting place for the location of relief supplies warehouse evaluated on the five criteria, $X_{6}$ would be accorded the next highest priority to be developed, and $X_{3}$ is indicated to be the worst one chosen in the evaluation.

\section{Conclusions}

Our study mainly discusses the selection of relief supplied warehouse location. A flexible and systematic framework 


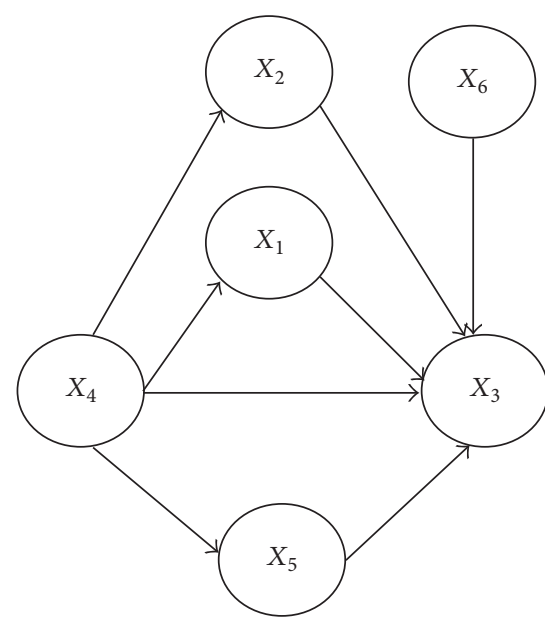

(a) Initial upward strong digraph

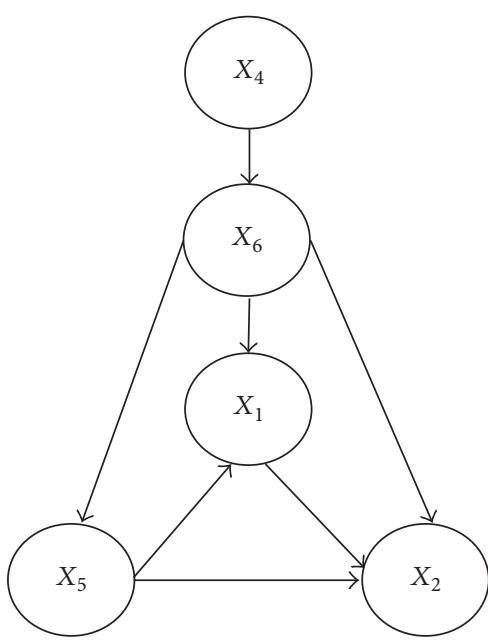

(b) Initial upward weak digraph

Figure 2

is provided to select the prepositioning warehouse location. Then, the key criteria used to evaluate the alternative locations are identified according to the different perspectives from both the literature and practical investigation. Additionally, we attempt to propose a new multicriteria ranking method in which preferences of the DM need not be expressed explicitly or implicitly in advance. Based on exploring stochastic pairwise dominant probability among alternatives, the proposed method can give explicit ranks of alternatives. The whole processes for applying the proposed method are unambiguous so that it is pretty acceptable to the DMs when making those hard decisions.

In the illustrative example, it is shown that our proposed method is effective and straightforward for obtaining an explicit ranking of alternatives under the condition that little weight information is available and the preference information of DMs is partially or completely missing. This brings us to believe that the proposed method in this paper can be adopted to deal with the problems of locating relief supplies warehouse and the results of the analysis will be clear and helpful to the DMs.

\section{Conflicts of Interest}

The authors declare that there are no conflicts of interest regarding the publication of this paper.

\section{Acknowledgments}

This research is supported by National Natural Science Funds of China (no. 71601062), MOE (Ministry of Education in China) Project of Humanities and Social Science (no. JS2016JYRW0076), Natural Science Funds of Anhui Province (no. 1708085QG165), National Key R\&D Plan (2016YFC0803203), and the Fundamental Research Funds for the Central Universities (nos. JZ2014HGBZ0730 and JZ2015HGBZ0481).

\section{References}

[1] M. S. Habib and B. Sarkar, "An Integrated Location-Allocation Model for Temporary Disaster Debris Management under an Uncertain Environment," Sustainability, vol. 9, no. 5, p. 716, 2017.

[2] F. Yang, Q. Yuan, S. Du, and L. Liang, "Reserving relief supplies for earthquake: a multi-attribute decision making of China Red Cross," Annals of Operations Research, vol. 247, no. 2, pp. 759785, 2016.

[3] M. Kress, "Humanitarian Logistics," in Operational Logistics, Management for Professionals, pp. 137-144, Springer International Publishing, Cham, 2016.

[4] B. M. Beamon and B. Balcik, "Performance measurement in humanitarian relief chains," International Journal of Public Sector Management, vol. 21, no. 1, pp. 4-25, 2008.

[5] B. Balcik and B. M. Beamon, "Facility location in humanitarian relief," International Journal of Logistics Research and Applications, vol. 11, no. 2, pp. 101-121, 2008.

[6] S.-Y. Roh, H.-M. Jang, and C.-H. Han, "Warehouse location decision factors in humanitarian relief logistics," Asian Journal of Shipping and Logistics, vol. 29, no. 1, pp. 103-120, 2013.

[7] S. Roh, The pre-positioning of humanitarian aid: the warehouse location problem [Doctoral, thesis], Section of Cardiff Business School, Cardiff University, 2012.

[8] G. Kovács and K. Spens, "Identifying challenges in humanitarian logistics," International Journal of Physical Distribution and Logistics Management, vol. 39, no. 6, pp. 506-528, 2009.

[9] P. Scott-Bowden, “The World Food Programme: Augmenting logistics," Forced Migration Review, vol. 18, pp. 17-19, 2003.

[10] L. N. Van Wassenhove, "Humanitarian aid logistics: supply chain management in high gear," Journal of the Operational Research Society, vol. 57, no. 5, pp. 475-489, 2006.

[11] M. Kuo, "Optimal location selection for an international distribution center by using a new hybrid method," Expert Systems with Applications, vol. 38, no. 6, pp. 7208-7221, 2011.

[12] Y. Li, X. Liu, and Y. Chen, "Selection of logistics center location using Axiomatic fuzzy set and TOPSIS methodology in logistics management," Expert Systems with Applications, vol. 38, no. 6, pp. 7901-7908, 2011. 
[13] S. Önüt, T. Efendigil, and S. S. Kara, "A combined fuzzy MCDM approach for selecting shopping center site: an example from Istanbul, Turkey," Expert Systems with Applications, vol. 37, no. 3, pp. 1973-1980, 2010.

[14] C. Kahraman, D. Ruan, and I. Doğan, "Fuzzy group decisionmaking for facility location selection," Information Sciences, vol. 157, no. 1-4, pp. 135-153, 2003.

[15] T. Demirel, N. Ç. Demirel, and C. Kahraman, "Multi-criteria warehouse location selection using Choquet integral," Expert Systems with Applications, vol. 37, no. 5, pp. 3943-3952, 2010.

[16] P. Alberto, "The Logistics of Industrial Location Decisions: An Application of the Analytic Hierarchy Process Methodology," International Journal of Logistics Research and Applications, vol. 3, no. 3, pp. 273-289, 2000.

[17] M. S. Kuo, G. H. Tzeng, and W. C. Huang, "Group decisionmaking based on concepts of ideal and anti-ideal points in a fuzzy environment," Mathematical and Computer Modelling, vol. 45, no. 3-4, pp. 324-339, 2007.

[18] J. Korpela and M. Tuominen, "A decision aid in warehouse site selection," International Journal of Production Economics, vol. 45, no. 1-3, pp. 169-180, 1996.

[19] N. U. Handayani, D. I. Rinawati, and Y. K. Wiguna, "Model of pre-positioning warehouse logistics for disaster eruption of Mount Merapi in Sleman Yogyakarta," in Proceedings of the 2015 Joint International Conference on Electric Vehicular Technology, ICEVT 2015 and Industrial, Mechanical, Electrical and Chemical Engineering, IMECE 2015, pp. 401-405, Indonesia, November 2015.

[20] T. Özcan, N. Çelebi, and Ş. Esnaf, "Comparative analysis of multi-criteria decision making methodologies and implementation of a warehouse location selection problem," Expert Systems with Applications, vol. 38, no. 8, pp. 9773-9779, 2011.

[21] Y. Kayikci, "A conceptual model for intermodal freight logistics centre location decisions," Procedia: Social and Behavioral Sciences, vol. 2, no. 3, pp. 6297-6311, 2010.

[22] R. Benayoun, B. Roy, and B. Sussman, "ELECTRE: Une méthode pour guider le choix en présence de points de vue multiples," Note de travail, vol. 49, 1966.

[23] B. Roy and P. Bertier, "La méthode ELECTRE II," Note de travail, vol. 142, 1971.

[24] B. Roy, "ELECTRE III: un algorithme de classements fondé sur une repré sentation floue des préférences en préferences en presence de critéres multiples," Cahiers du Centre d'Etudes de Recherche Operationelle, vol. 20, no. 1, pp. 3-24, 1978.

[25] B. Roy and J. C. Hugonnard, "Ranking of suburban line extension projects on the Paris metro system by a multicriteria method," Transportation Research Part A: General, vol. 16, no. 4, pp. 301-312, 1982.

[26] W. Yu, "ELECTRE TRI: aspects méthodologiques et manuel d'utilisation. Document- Université de Paris-Dauphine," LAMSADE, 1992.

[27] B. Roy and D. Bouyssou, Aide multicritère à la décision: Méthodes et cas, Economica, Paris, France, 1993.

[28] A. Karagiannidis and N. Moussiopoulos, "Application of ELECTRE III for the integrated management of municipal solid wastes in the greater Athens area," European Journal of Operational Research, vol. 97, no. 3, pp. 439-449, 1997.

[29] L. Duckstein and M. Gershon, "Multicriterion analysis of a vegetation management problem using ELECTRE II," Applied Mathematical Modelling, vol. 7, no. 4, pp. 254-261, 1983.
[30] M. Beccali, M. Cellura, and D. Ardente, "Decision making in energy planning: The electre multicriteria analysis approach compared to a fuzzy-sets methodology," Energy Conversion and Management, vol. 39, no. 16-18, pp. 1869-1881, 1998.

[31] B. Roy and D. Bouyssou, "Comparison of two decision-aid models applied to a nuclear power plant siting example," European Journal of Operational Research, vol. 25, no. 2, pp. 200-215, 1986.

[32] B. Roy, "The outranking approach and the foundations of electre methods," Theory and Decision, vol. 31, no. 1, pp. 49-73, 1991.

[33] K. Govindan and M. B. Jepsen, "ELECTRE: a comprehensive literature review on methodologies and applications," European Journal of Operational Research, vol. 250, no. 1, pp. 1-29, 2016.

[34] J. Figueira, S. Greco, and M. Ehrogott, Multiple criteria decision analysis: State of the art surveys, Springer, New York, USA, 2005.

[35] M. R. Mahmoud and L. A. Garcia, "Comparison of different multicriteria evaluation methods for the Red Bluff diversion dam," Environmental Modeling and Software, vol. 15, no. 5, pp. 471-478, 2000.

[36] T. Tervonen, J. Figueira, R. Lahdelma, and P. Salminen, An inverse approach for ELECTRE III. Research Report 20/2004, the Institute of Systems Engineering and Computers (INESCCoimbra), Coimbra, Portugal, 2004.

[37] J. Hokkanen, R. Lahdelma, K. Miettinen, and P. Salminen, "Determining the implementation order of a general plan by using a multicriteria method," Journal of Multi-Criteria Decision Analysis, vol. 7, no. 5, pp. 273-284, 1998.

[38] R. Lahdelma and P. Salminen, "Pseudo-criteria versus linear utility function in stochastic multi-criteria acceptability analysis," European Journal of Operational Research, vol. 141, no. 2, pp. 454-469, 2002.

[39] H. Zhou, J. Wang, and H. Zhang, "Stochastic multicriteria decision-making approach based on SMAA-ELECTRE with extended gray numbers," International Transactions in Operational Research, 2017.

[40] T. Tervonen, R. Lahdelma, J. A. Dias, J. Figueira, and P. Salminen, "SMAA-TRI: a parameter stability analysis method for ELECTRE-TRI," in In Environmental Security in Harbors and Coastal Areas, G. A. Kiker and I. Linkov, Eds., pp. 217-231, Springer, Berlin, 2007.

[41] J. Hokkanen, P. Salminen, E. Rossi, and M. Ettala, "The choice of a solid waste management system using the Electre II decisionaid method," Waste Management \& Research, vol. 13, no. 2, pp. 175-193, 1995.

[42] B. Roy and P. Vincke, "Multicriteria analysis: survey and new directions," European Journal of Operational Research, vol. 8, no. 3, pp. 207-218, 1981.

[43] D. Jun, F. Tian-Tian, Y. Yi-Sheng, and M. Yu, "Macro-site selection of wind/solar hybrid power station based on ELECTRE-II," Renewable \& Sustainable Energy Reviews, vol. 35, pp. 194-204, 2014.

[44] R. Lahdelma, J. Hokkanen, and P. Salminen, "SMAA—stochastic multiobjective acceptability analysis," European Journal of Operational Research, vol. 106, no. 1, pp. 137-143, 1998.

[45] R. Lahdelma and P. Salminen, "SMAA-2: stochastic multicriteria acceptability analysis for group decision making," Operations Research, vol. 49, no. 3, pp. 444-454, 2001. 


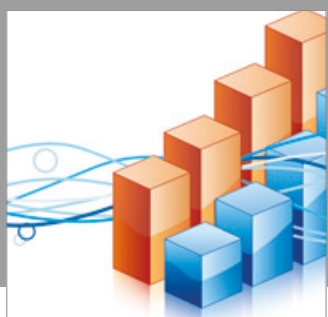

Advances in

Operations Research

vatersals

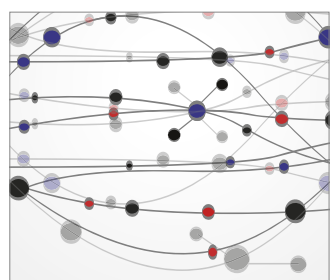

\section{The Scientific} World Journal
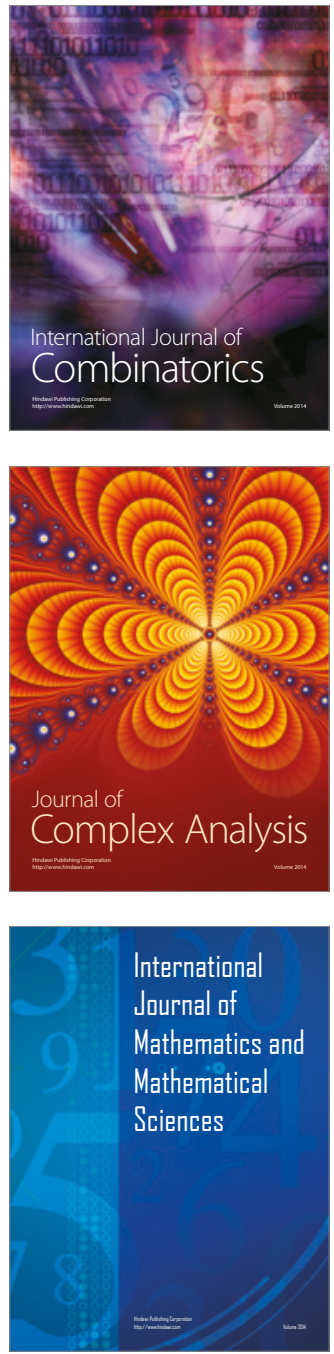
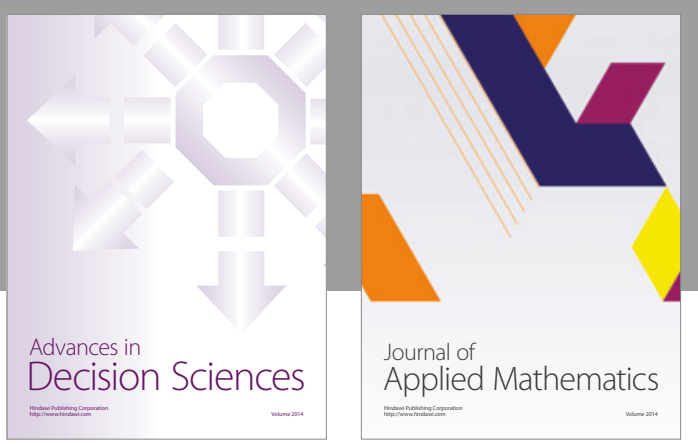

Algebra

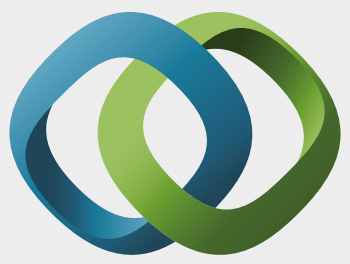

\section{Hindawi}

Submit your manuscripts at

https://www.hindawi.com
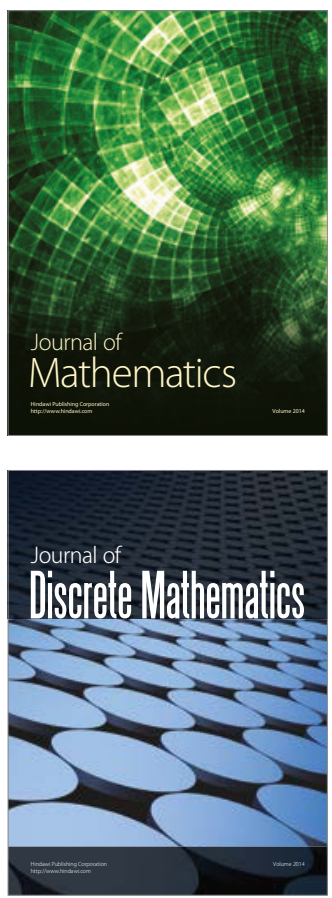

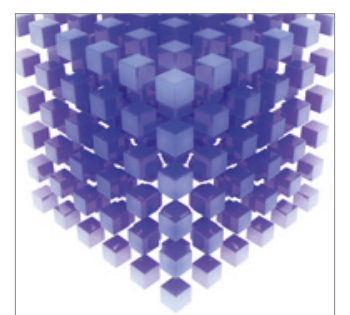

Mathematical Problems in Engineering
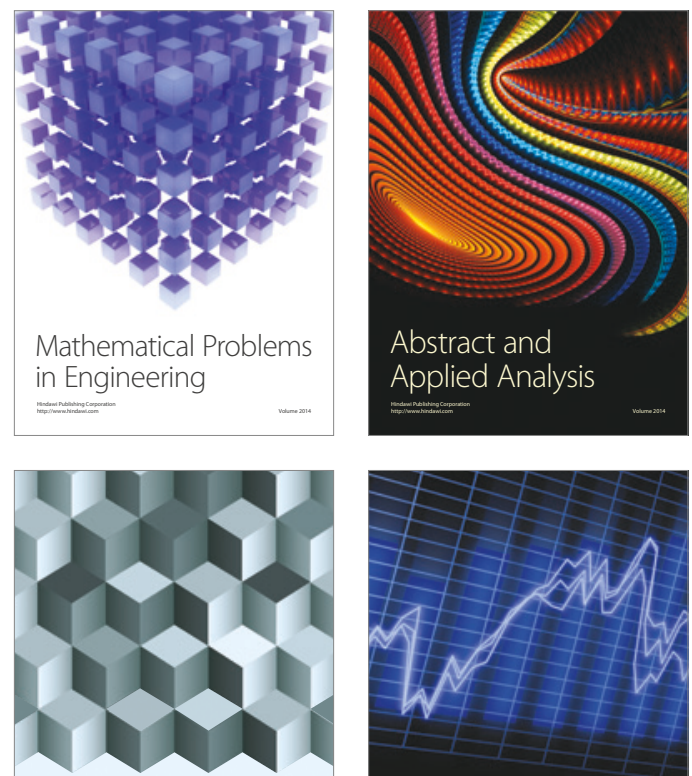

Journal of

Function Spaces

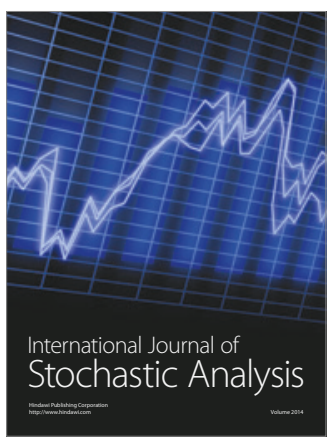

Probability and Statistics
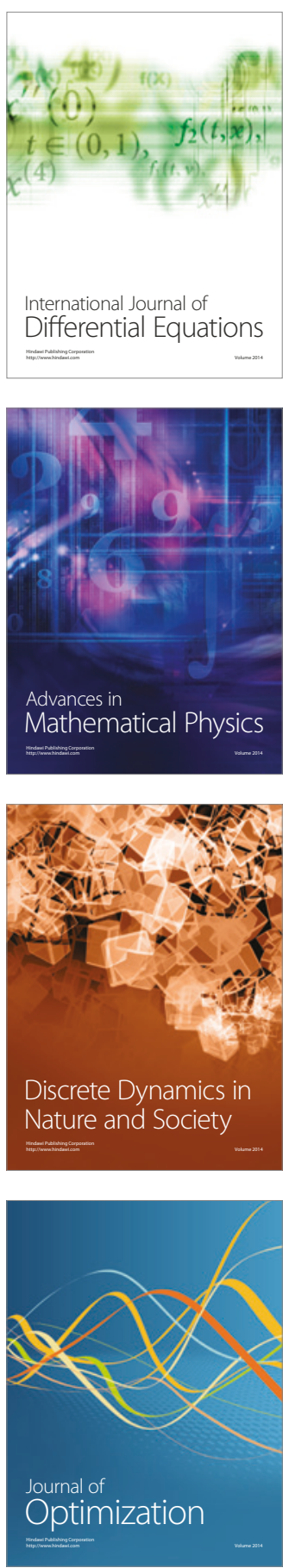